\title{
Fabrication of Microlens Array and Its Application: A Review
}

\author{
Wei Yuan ${ }^{1,2}$, Li-Hua Li $i^{1,2^{*}} \mathbb{B}$, Wing-Bun Lee ${ }^{1,2}$ and Chang-Yuen Chan ${ }^{1,2}$
}

\begin{abstract}
Microlens arrays are the key component in the next generation of 3D imaging system, for it exhibits some good optical properties such as extremely large field of view angles, low aberration and distortion, high temporal resolution and infinite depth of field. Although many fabrication methods or processes are proposed for manufacturing such precision component, however, those methods still need to be improved. In this review, those fabrication methods are categorized into direct and indirect method and compared in detail. Two main challenges in manufacturing microlens array are identified: how to obtain a microlens array with good uniformity in a large area and how to produce the microlens array on a curved surface? In order to effectively achieve control of the geometry of a microlens, indirect methods involving the use of 3D molds and replication technologies are suggested. Further development of ultraprecision machining technology is needed to reduce the surface fluctuation by considering the dynamics of machine tool in tool path planning. Finally, the challenges and opportunities of manufacturing microlens array in industry and academic research are discussed and several principle conclusions are drawn.
\end{abstract}

Keywords: Microlens array, Ultraprecision machining, 3D image system, MEMS

\section{Introduction}

Natural compound eyes are extensively prominent in the biological optical systems of many diurnal insects or deep-water crustaceans, and such eyes consist of a mosaic of hexagonal ommatidia that work as tiny optical units [1-4]. Unlike single aperture eyes, natural compound eyes are characterized as having extremely large field of view angles, low aberration and distortion, high temporal resolution and infinite depth of field [3, 4]. However, the compound eye image system has intrinsic low resolution and sensitivity [4]. The image resolution is subject to both the number and size of the ommatidia. If the image resolution of compound eyes increases to the same level as the human aperture eye, the radius of the overall lens would be at least 1 meter [5].

Although the image resolution and sensitivity of compound eyes are relatively low, microlens arrays, the artificial counterpart of natural compound eyes, still have

\footnotetext{
*Correspondence: lihua.li@polyu.edu.hk

${ }^{2}$ Shenzhen Branch of State Key Laboratory of Ultra-precision Machining Technology, PolyU Shenzhen Research Institute, Shenzhen 518000, China Full list of author information is available at the end of the article
}

crucial potential in a variety of applications in image systems, under the condition that high-resolution is not always required. For example, microlens array are more suitable in the extremely miniaturized imaging systems and 3D light field cameras [6]. In addition, the high quality microlens arrays were applied in color imaging systems, 3D image acquisition systems and fingerprint identification systems [7-9].

Since the 1980s, the fabrication of microlens array is realized by different methods, such as Micro-electromechanical Systems (MEMS) based technologies [10-17] and ultraprecision machining technologies [18-20]. However, little work has been focused on the comparison of these method in terms of the surface finish, form error and the efficiency of production. One of the major challenge in the fabrication of the microlens array is the fabrication and assembly accuracy in a large area [19-21]. As the image resolution of a compound eye optical system is increased with the number of microlens and the radius of each microlens unit, enlarging the overall size of a microlens array can make up the deficiency. However, to achieve the required uniformity in a large area is very difficult [19]. Another challenge for microlens fabrication is
SpringerOpen

(c) The Author(s) 2018. This article is distributed under the terms of the Creative Commons Attribution 4.0 International License (http://creativecommons.org/licenses/by/4.0/), which permits unrestricted use, distribution, and reproduction in any medium, provided you give appropriate credit to the original author(s) and the source, provide a link to the Creative Commons license, and indicate if changes were made. 
producing microlens array on a flexible layer or a curved surface. The curved artificial compound eye is similar to the eye of the fruit fly Drosophila, which is more compatible and has a larger Field of View (FOV) [22]. Such curved compound eye imaging systems may have great potential in terrestrial aerial vehicles, visual reality systems, surveillance etc. Image detectors, such as conventional complementary metal-oxide-semiconductor (COMS) and charge-coupled device (CCD), are planar and not suitable for curved image systems. Recent developments [23] in flexible technologies enable the formation of microlens arrays on flexible substrates which are bent to a spherical surface. Similar to the problem in the fabrication of planar compound eye, the requirement of precise alignment of the photodetector and microlens is hard to achieve.

In the light of the above, this paper aims to review the latest research on the progress of microlens array fabrication technologies. In Section 2, the operation principle of the compound eye is briefly introduced to provide background for the design of microlens array. In Section 3, the state-of-art technologies, including the direct and indirect methods for fabricating microlens array are reviewed and compared. Section 4 describes the applications of microlens array. Finally, the challenges and opportunities of manufacturing microlens arrays in industry and academic research are discussed and several principle conclusions are drawn in Section 5.

\section{Principle of Compound Eyes}

In nature, compound eyes can be categorized into 2 types, e.g., apposition compound eyes and superposition compound eyes, as shown in Figure 1. In natural apposition compound eyes, the light through each ommatidia is received by only one photo receptor [1,2]. In contrast, every photo receptor in the superposition compound eye is able to acquire light from several ommatidia. Therefore, superposition eyes are much more light-sensitive, and more suitable for deep-water crustaceans living in dim light. However, the main drawbacks of superposition eyes are the aberrations as the consequence of the combination of light from different ommatidia. Therefore, the artificial compound eyes are mainly of the apposition form.

As shown in Figure 1(a), in nature, the ommatidia of apposition compound eyes are arranged on a curved surface of radius $R_{e}$ and the receptors of diameter $d$ are distributed on the focal points of the ommatidia. The geometric size of each ommatidia is denoted by the pitch $D$ and focal length $f$. The acceptance angle $\Delta \varphi$ and interommatidial angle $\Delta \Phi$ can be expressed as follows [24]:

$$
\Delta \varphi=\sqrt{\left(\frac{d}{f}\right)^{2}+\left(\frac{\lambda}{D}\right)^{2}},
$$
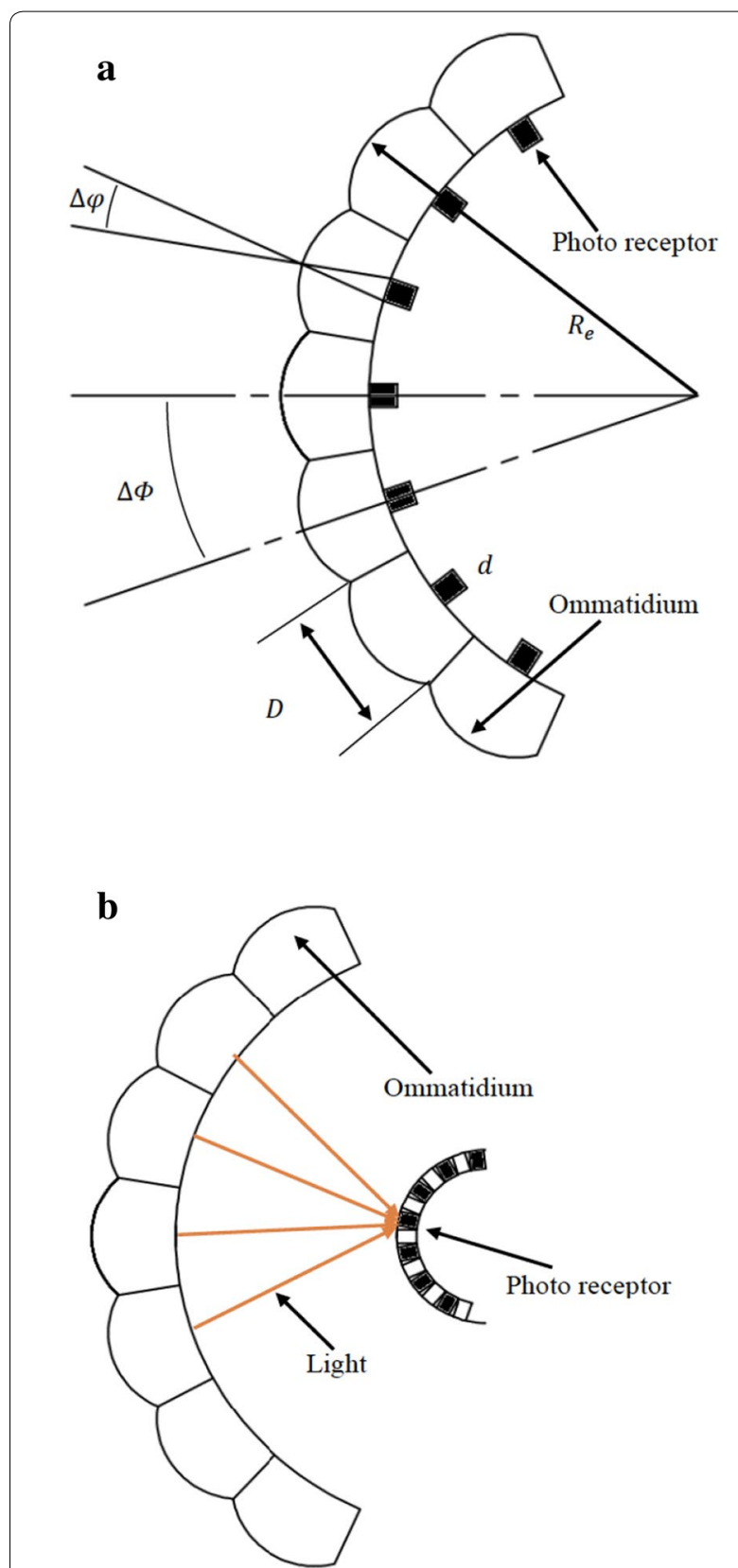

Figure 1 Natural compound eyes: (a) apposition compound eyes, (b) superposition compound eyes

$$
\Delta \Phi=\frac{D}{R_{e}},
$$

where $\lambda / D$ is the full width maximum of the Gaussian approximation of the Airy function.

In most of artificial compound eye optical systems, microlens array which is the counterpart of ommatidia, is arranged on a plane (Figure 2) to fit with CCD and 


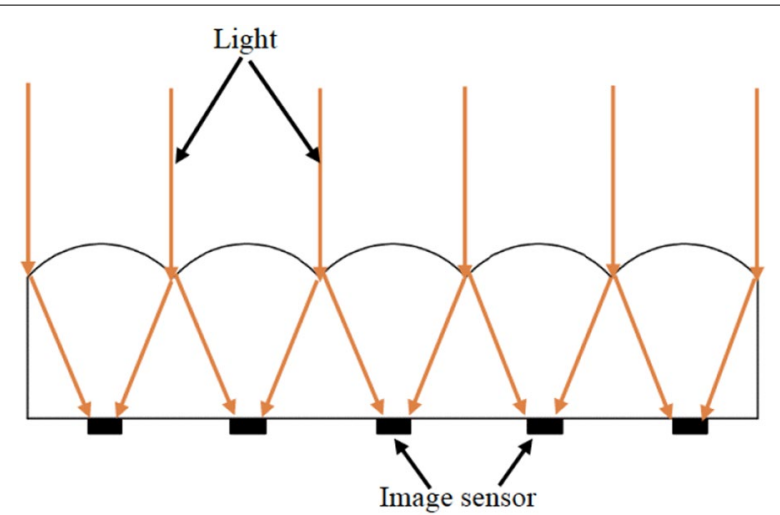

Figure 2 Artificial compound eye optical systems with planar structure

CMOS. Moreover, the fabrication process of microlens array on a plane is much simpler.

For each unit of the microlens array, the geometric size is determined by the pitch $(D)$, the height $(h)$, the radius of curvature $\left(R_{\mathrm{u}}\right)$ and contact angle $(\theta)$, shown in Figure 3 . These parameters can be measured through optical microscopy, scanning electron microscopy as well as contact profilometry. The quality of the microlens often is denoted by the numerical aperture $(N A)$, surface roughness and array uniformity.

The numerical aperture is calculated as [24]:

$$
N A=n \cdot \sin \alpha,
$$

where $n$ is the refractive index of the medium between the object and the microlens. The half aperture angle $\alpha$

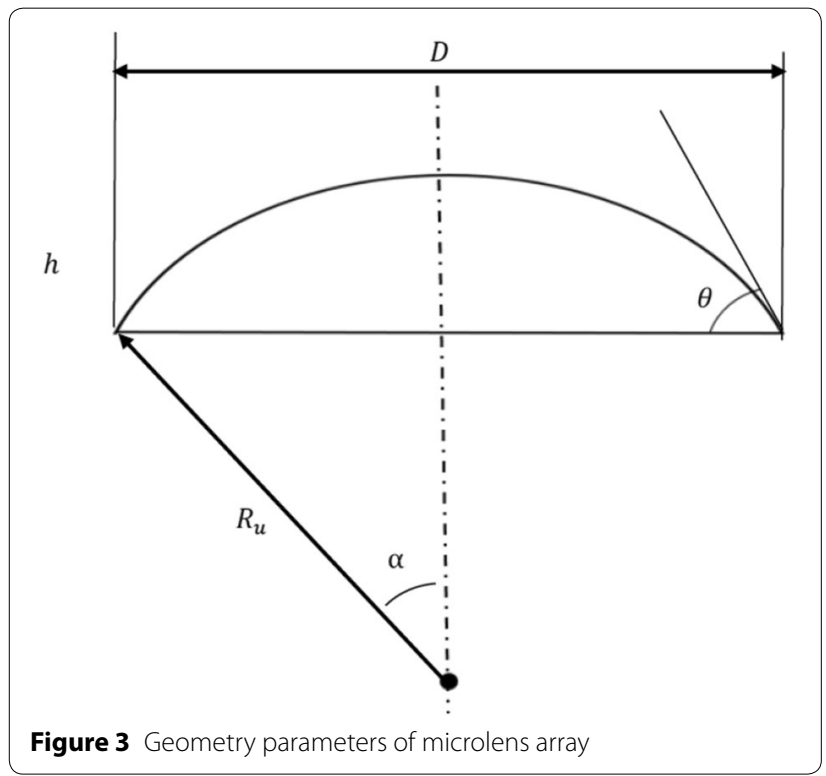

can be obtained from the height $(h)$, the radius of curvature $\left(R_{u}\right)$ :

$$
\alpha=\arccos \left(\frac{R_{u}-h}{R_{u}}\right) .
$$

With the increase of the value of $N A$, the resolution and magnification of the microlens also increases. The contact angle $\theta$ is equal to half aperture angle $\alpha$ and the F-number $F^{\#}$ is defined as [25]:

$$
F^{\#}=\frac{1}{2 n \cdot \sin \alpha} \text {. }
$$

Surface roughness is another important parameter in evaluating the optical performance of a lens. And it is strongly affected by the fabrication process. Optics with large surface roughness may suffer from scattering issues, decreasing the efficiency of the contrast and light collection [26]. The array uniformity is of great importance in the imaging system, especially when the area of microlens array is large, and light retrace is needed for further process. The array uniformity can be described by the standard deviation of the height and the radius of curvature [26].

\section{Fabrication Methods}

The fabrication methods for microlens arrays are categorized into direct methods and indirect methods. The direct method does not need to fabricate a mask or a mold insert with concave 3D microstructures. The shape of microlens is usually formed based on the surface tension effect when the material is in a thermoplastic state or liquid state resulting a super smooth surface (arithmetic average roughness $R_{a}$ less than $1 \mathrm{~nm}$ ) [27-29]. More importantly, these methods involve simple and cost-effective processes, which are preferred in industry. However, it is still very difficult to control the microlens precision because the geometry of the microlens is only determined by the controlling parameters such as temperature, wettability, pressure and process time. The indirect methods need to fabricate the mold with concave microlenses and produce the final lenses by replication technologies, such as hot embossing, compact molding and injection molding. Using the indirect method, the shape of microlens array can be well-controlled but the process is complex.

\subsection{Direct Fabrication Methods}

\subsubsection{Thermal Reflow Method}

The thermal reflow method has been used to produce microlens arrays in the last few decades [30-34]. The fabrication process is depict in Figure 4 [30]: first, the photoresist layer is coated onto the substrate to let the UV-light thrust through the mask which has circular 


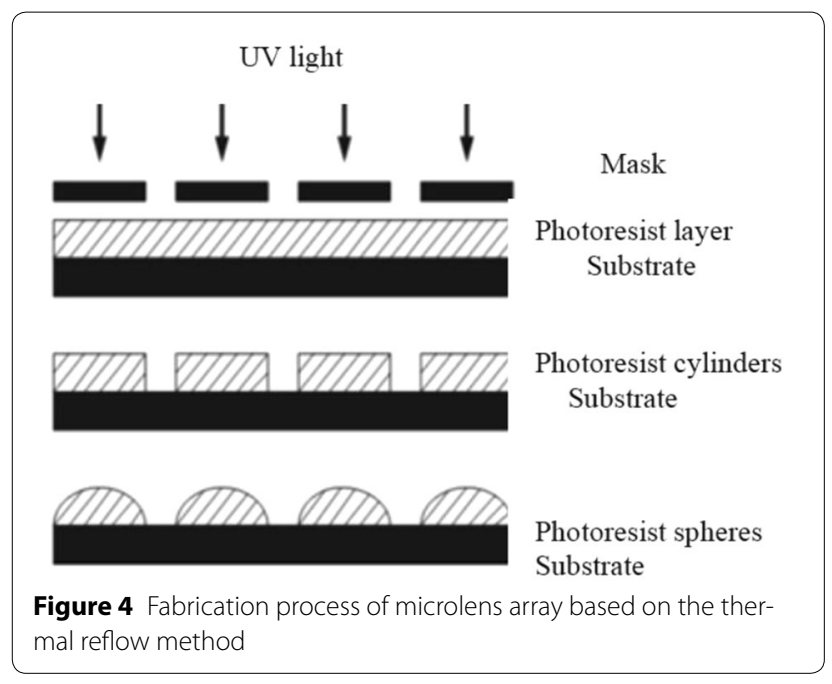

array patterns. Second, the photo-resist layer is developed and the cylindrical isolated-islands are then generated. Third, the isolated-islands are heated to a certain temperature, then the cylindrical island will turn into a spherical structure due to the effect of surface tension. In this method, an optical separator is not needed and the microlens array is fabricated by the common MEMS technique. However, the contact angle is hard to control because it is only affected by the wettability of both the material on the substrate and the surrounded air around the microlens, rather than the size of the isolated-islands.

\subsubsection{Microplastic Embossing Method}

The microplastic embossing method is a low-cost and highly efficient technique, which was developed to the fabrication of plastic microlens [29, 35-37]. The partial-filling technology [37] based on the microplastic embossing process does not require a mold insert with the desired microlens geometry, and thereby alleviates the surface defects induced by contact between mold insert and injected materials. Based on this method, a silicon mold insert of circular openings with a few hundred micrometers is first fabricated by a deep reactive ion etching process. Then a polymer substrate is placed between the heating plates and the silicon mold insert, as shown in Figure 5. The microlenses are constructed by applying an external pressure at an elevated temperature above the glass transition point for a given time. Finally, the newly formed microlens is cooled down by decreasing the temperature as slowly as possible to reduce the thermal stress and attendant replication errors. The temperature-dependent viscosity and surface tension are the major factors in the fabrication process, determining both the height and radius of the microlens. The applied pressure has a linear relationship with the height of the

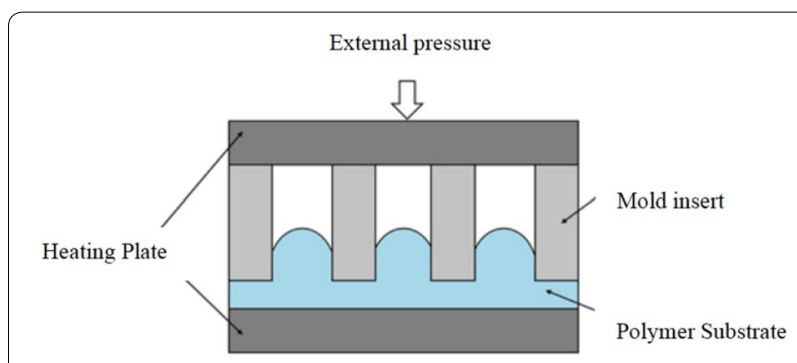

Figure 5 Fabrication process of microlens array based on microplastic hot embossing

microlens but has little effect on the radius. The processing temperature is capable of affecting both the radius and height in a complicated relationship. The microplastic embossing method is considered as a one-step molding process, and is not influenced by the quality of the mold surface [35].

\subsubsection{Microdroplet Jetting Method}

Microdroplet jetting, also known as ink jet printing, is another direct method for fabricating microlenses [38-42]. Figure 6 shows a schematic diagram of the working principle of this method [39]. The droplets of a UV-polymerizable liquid are ejected from a nozzle to a substrate. When the droplets reach this substrate, they are exposed to UV light and converted to a solid state with a super smooth shape. In order to obtain a microlens with large a NA (more than 0.4), the substrate is treated by Nano texturing and fluorodecyltrichlorosilane (PFTS) or $\mathrm{C}_{4} \mathrm{~F}_{8}$ coating [39-41]. This method is suitable for fabricating microlens arrays in a large area rapidly at room temperature. However, it is hard to control the consistency and feature size of the microlens.

\subsection{Indirect Method}

In contrast with direct methods for the fabrication of compound eye microlens arrays, indirect methods need

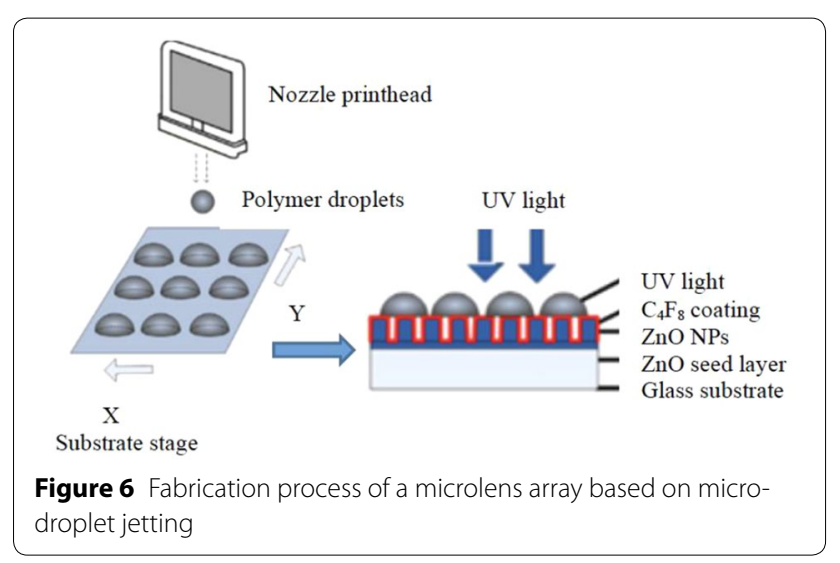


a mold with a concave spherical microlens. The final microlens array is produced by replication technologies such as injection molding, hot embossing or UV molding. The key to the indirect methods is how to generate concave microlenses with precise geometry. The potential technologies are divided into two categories, i.e., MEMS based technologies and ultraprecision machining technologies.

\subsubsection{MEMS Based Methods}

The standard MEMS methods utilizes photolithography to generate patterns on the mask layer and chemical reactions to etching the curvature of microlens onto the substrate [40, 43-48]. Albero et al. [43] proposed a novel microlens wafer-scale fabrication method based on isotropic wet etching technology, shown in Figure 7. First, the protection layers of $\mathrm{SiO}_{2}, \mathrm{Si}_{3} \mathrm{~N}_{4}$ and $\mathrm{NiCr}$ are coated on the substrate. Then the patterns are generated though photolithography, reactive ion etching (RIE) and the HF solution step by step. After that, the wafers are immersed in the isotropic etch solution to generate the concave microlenses. Finally, the mold is finished after the removal of the mask layer. A wide range of lens geometries and lens arrays with good surface smoothness, high uniformity and repeatability can be achieved using on this method. However, this approach requires expensive equipment and complicated procedures to produce the mask on the wafer. Besides, the control precision of the mask fabrication technology must be improved to meet the requirements in the miniaturization of lenses.

To overcome this problem, direct writing technologies have been developed. For example, femtosecond laser wet etching was developed in which the patterns are directly generated on the wafer by laser processing. Then,

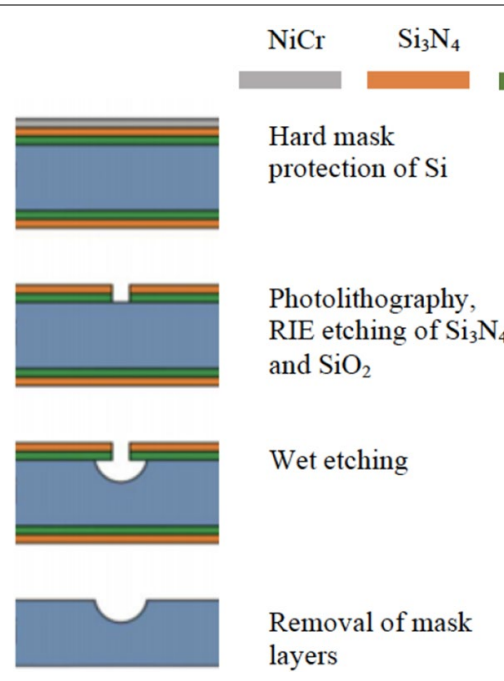

Figure 7 Fabrication process by standard MEMS technologies in the wet etching process, the concave microlens are formed as the induced region has a higher selective etching rate than the other parts. Other examples of direct writing techniques includes focused ion beam writing $[47,48]$ and electron beams writing $[49,50]$.

Direct laser writing can be used to produce patterns on a spherical substrate. Curved artificial compound eyes (CACEs) are realized by this method [51]. However, the optical system is hard to be miniaturized because it needs overlapping of the concave and the convex bulk lenses (the radius of the spherical lenses is $40 \mathrm{~mm}$ ). Another approach to fabricate the CACEs is by transforming the 2D microlens-pattern films into a 3D shape by the negative pressure deformation process [52-55]. However, the uniformity of the microlenses need to be improved because in the negative pressure deformation process, some microlens at the marginal areas may be damaged [55].

\subsubsection{Ultraprecision Machining Methods}

Ultraprecision machining technologies, such as diamond micro-milling and single point diamond turning (SPDT) are effective methods to fabricate microstructures and nanostructures with good uniformity in a large area [18, 21, 56-58]. Such ultraprecision technologies are integrated into the process chain for mass fabrication of microlens arrays. Ball-ending milling usually utilizes half-arc single crystal diamond tools to removal metallic materials [56, 57]. Metallic materials such as OFHC-Cu, $\mathrm{AlMg}_{3}$ and NiP can be processed. The achieved surface roughness $\left(R_{\mathrm{a}}\right)$ is as low as $5 \mathrm{~nm}$. However, this method needs to machine the microlenses one by one, which severely extends the operation time and increases the cost. To our knowledge, the bottom of the each concave microlens is affected by the alignment error (around 1 $\mu \mathrm{m})$ between the vertex of the cutting edge and the spindle axis. With regard to this, the single point diamond turning (SPDT) method is performed to produce microlens arrays with high quality by a slow slide servo $[18,19$, $58]$ or a fast tool servo $[21,59,60]$.

The procedures of single point diamond turning of microlens arrays on a flat surface were described by Zhang et al. [20]. A design model is first generated based on the ideal structures of microlens array. Then the ideal tool path is calculated based on the design model and cutting parameters such as spindle speed $(S)$ and feedrate $(F)$ based on the cylindrical coordinate method $[18,19]$. After that, the final tool path is modified by considering alignment error, tool radius error and squareness error. Two translation axes $(X$ and $Z)$ and a rotational spindle axis $(C)$ are precisely controlled to generate the 3D structures on the cylindrical end face of the workpiece. The form errors of the microlens array influence the 


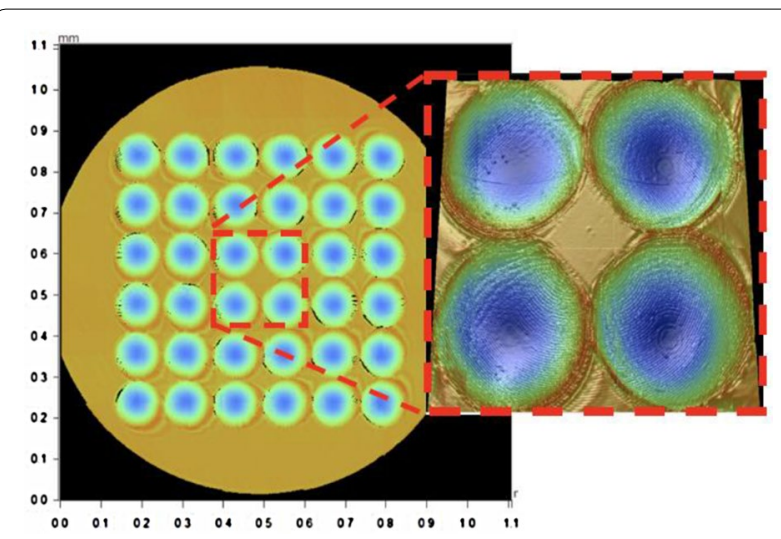

Figure 8 Surface fluctuation on the cutting parts around the inflection points of path measured by Laser Interferometers

machining errors. However, the surface fluctuation (Figure 8 ) is probably noticeable on the cutting parts around the inflection points of the path, which reduces the optical performance severely. The surface fluctuation appears due to the limit of the machine dynamic response. With regard to this, a new fabrication method is proposed to cut the microlens on a metal sheet mounted on the side face of a cylindrical basement, as shown in Figure 9. After the turning process, the metal sheet is striped off and inserted in the injection mold. Then the microlenses are produced by microinjection molding process. Based on this method, the surface fluctuation is reduced and a smoother surface is obtained, with the surface roughness $\left(R_{\mathrm{a}}\right)$ less than $10 \mathrm{~nm}$ and the PV-value less than 0.150 $\mu \mathrm{m}$ [61]. One of the drawbacks of this method is that, in order to avoid the interference between the clearance face of diamond tool and the machined concave microlens, a small F number (less than 4) is difficult to achieve. It is expected that the surface finish will be further improved by adaptive tool path planning, considering the dynamic response of the machine tool. Some improvement has been achieved by developing the quasi-elliptical tool servo (QETS) technique when producing a single microlens on the mold [62].

Ultraprecision machining was also applied to fabricate $3 \mathrm{D}$ compound eye lens arrays $[63,64]$. It was reported that 601 individual compound eye microlenses (aperture of $0.58 \mathrm{~mm}$ ) and the related microprisms were produced in a $20 \mathrm{~mm}$ diameter area, providing a large light deviation angle of $18.43^{\circ}$ and maximal FOV of $180^{\circ}$, if the entire hemispherical surface is fabricated with microlenses. The microprism array and microlens array were precisely fabricated on a curved and a flat surface respectively, with a combination of single point diamond turning, diamond broaching and micromilling processes [63]. However, the intensity of the microlens on the hemispherical surface is

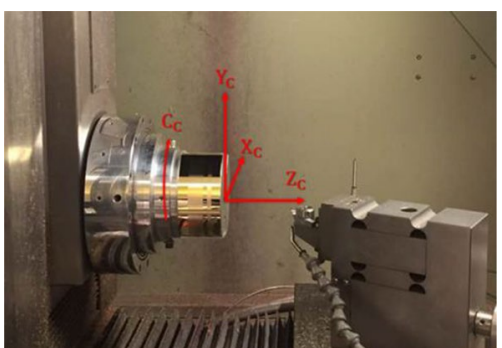

Figure 9 Turning of microlens array on the mental sheet

low, therefore the measured FOV is much smaller than the maximum theoretical value.

\section{Applications}

Microlens arrays can be integrated into a light field camera to achieve the function of "take photo and then focus" $[65,66]$ and obtain an image with both large FOV and aperture. Figure 10 shows the current light field of cameras, including the camera arrays developed by Stanford $[67,68]$; Integrated lens array designed by the Adobe company [69]; light field camera and microscope developed by Lytro [25, 70]; 3D light field camera produced by Raytrix [71] and compound eye camera module developed by the Toshiba company [72]. Compared with the camera array based devices, the microlens based devices have more potential in the market because of their light and compact structure. Such microlens array is mounted between the main lens and the light sensor, changing the light path (Figure 11). Figure 12 shows the experimental setup and the image acquired by the camera, having a compound eye microlens array fabricated by single point diamond turning and injection molding. Figure 12(b) shows the images processed by the digital refocus algorithm. To our knowledge, although 3D images can be obtained, however, the resolution of image obtained from

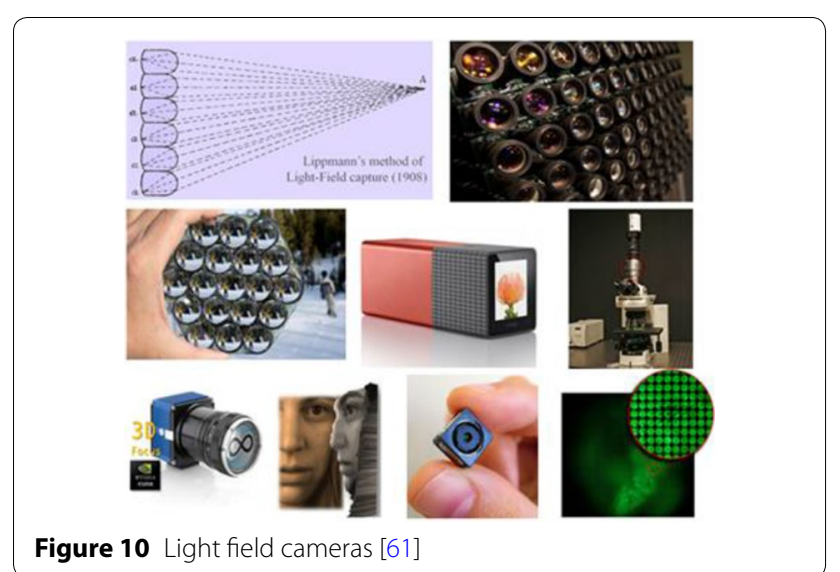




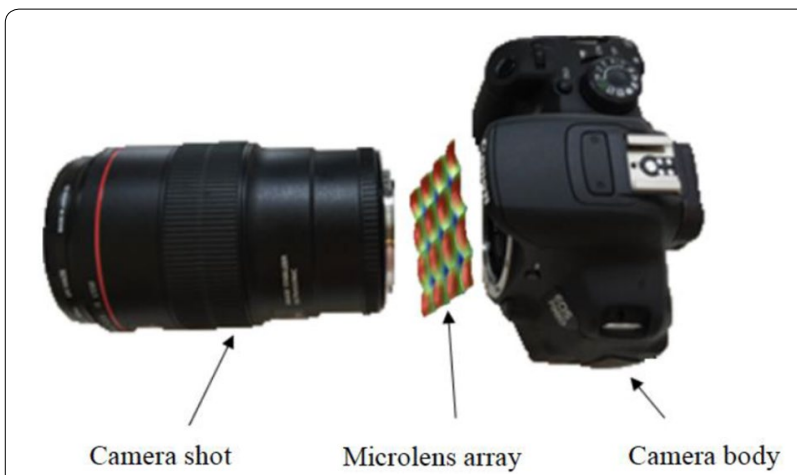

Figure 11 3D camera with compound eye microlens insert

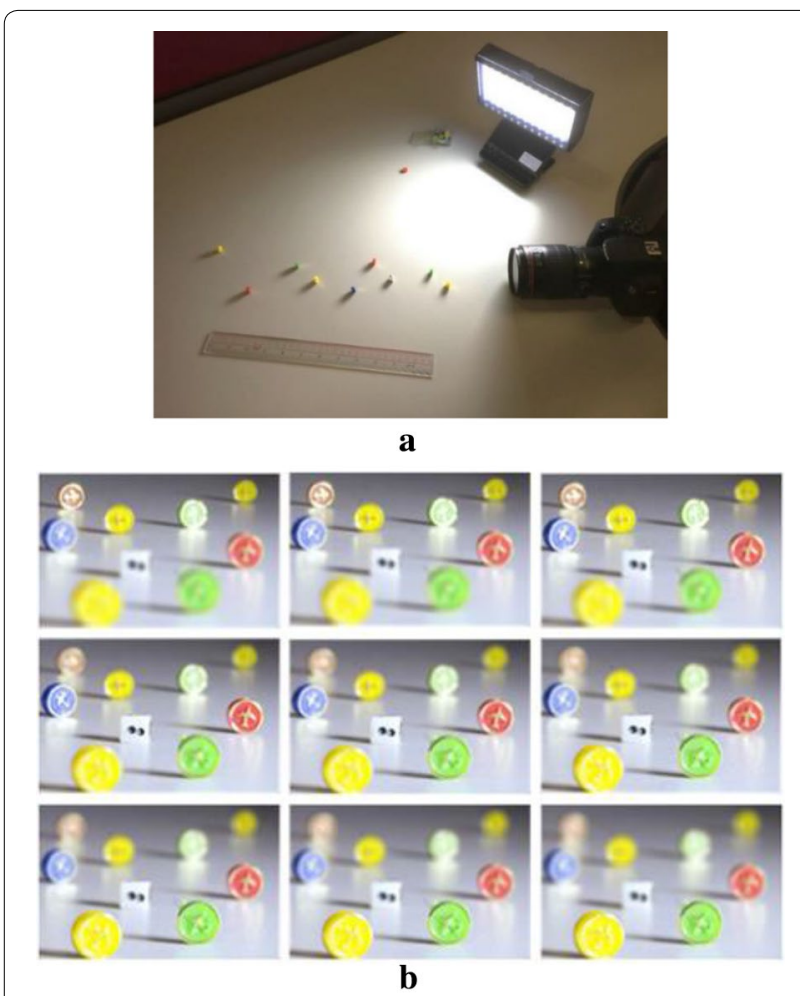

Figure 12 3D light field camera (a) experiment setup; (b) the image processed by digital refocus algorithm [61]

current light field camera (less than $1200 \times 900$ pixels) is much lower than the single-lens reflex camera.

\section{Conclusions and Outlook}

In this paper, the most important fabrication technologies for microlens arrays are reviewed and compared. By reviewing the advantages and disadvantages of those technologies, the following conclusions are drawn.
(1) The direct fabrication technologies, including the thermal reflow method, microplastic embossing and microdroplet jetting method, are simple and low-cost processes which are suitable for mass industrial production, but it is very difficult to control the accuracy of the microlenses shape based on the direct fabrication methods.

(2) Compared with MEMS based technologies, ultraprecision machining is more suitable in terms of producing microlens array on a mold with good uniformity in a large area. The shape of microlens can be well controlled by ultraprecision machining. Further improvement is needed to reduce the surface fluctuation and surface roughness by optimizing the tool path considering the dynamics of machine tools.

(3) Both the MEMS based technologies and ultraprecision machining are able to fabricate curved compound eye microlenses, but the production quality needs to improve.

(4) 3D imaging systems inserted with microlens array can be used to capture the light field information, but the spatial resolution is much lower compared with that of photos captured by 2D camera. These 3D imaging systems may be applied in the situation that the high spatial resolution is not required.

\section{Authors' contributions}

WY carried out the studies in the reviews of the principles of compound eye and the fabrication methods. He wrote the draft. LL investigated the applications of microlens array and she also contributed to the review of indirect fabrication method including molding process. WL and CC shared many fundamental ideas in the ultraprecision machining technologies, thermal reflow method and UV molding technology. CC conducted proof reading and made some critical revisions. All authors read and approved the final manuscript.

\section{Author details}

${ }^{1}$ State Key Laboratory of Ultra-precision Machining Technology, Partner Laboratory in the Hong Kong Polytechnic University, Hong Kong 999077, China.

${ }^{2}$ Shenzhen Branch of State Key Laboratory of Ultra-precision Machining Technology, PolyU Shenzhen Research Institute, Shenzhen 518000, China.

\section{Authors' Information}

Wei Yuan born in 1990, is currently a PhD candidate at State Key Laboratory of Ultra-precision Machining Technology, the Hong Kong Polytechnic University, China. He received his bachelor degree from Hefei University of Technology, China and master degree from The Chinese University of Hong Kong, China. His interests include metal cutting theory, ultraprecision machining processing and robotics. Li-Hua Li born in 1981, is current a research assistant at State Key Laboratory of Ultra-precision Machining Technology, the Hong Kong Polytechnic University, China. She earned her PhD degree in measurement science and technology from Tsinghua University, China, in 2012. Dr. Li's research focuses on the study of design theory of optical elements, fabrication and measurement technology of optics. Wing-Bun Lee born in 1951, is currently the Head of State Key Laboratory of Ultra-precision Machining Technology, the Hong Kong Polytechnic University, China. His teaching and research interests include advanced manufacturing technology, materials processing, ultra-precision machining, manufacturing strategy and knowledge management systems. Chang-Yuen Chan born in 1965, is current the project manager at State Key Laboratory of Ultra-precision Machining Technology, the Hong Kong Polytechnic University, China. He earned his PhD degree in mechanical engineering from Hong Kong University, China, in 1995. Dr. Chan's research focuses on the study of ultraprecision machining technology and 3D imaging processing. 


\section{Acknowledgements}

Supported by Shenzhen Science, Technology and Innovation Commission of China (Grant No. JCYJ20150630115257902), the Research Grants Council of the Hong Kong Special Administrative Region of China (Grant No. ITS/339/13FX), and Research Committee of The Hong Kong Polytechnic University, China (Grant No. RUKO).

\section{Competing Interests}

The authors declare that they have no competing interests.

\section{Ethics approval and consent to participate}

Not applicable.

\section{Publisher's Note}

Springer Nature remains neutral with regard to jurisdictional claims in published maps and institutional affiliations.

Received: 19 October 2016 Accepted: 14 January 2018

Published online: 27 February 2018

\section{References}

1. M F Land, D-E Nilsson. Animal eyes. Oxford University Press, 2012.

2. M F Land. The optics of animal eyes. Contemporary Physics, 1988, 29(5): 435-455.

3. G A Horridge. The separation of visual axes in apposition compound eyes. Philosophical Transactions of the Royal Society of London B: Biological Sciences, 1978, 285(1003): 1-59.

4. M F Land. Variations in the structure and design of compound. Facets of Vision, 1989, 3: 30-73.

5. YXu, S Yan, C Zhou, et al. Advances in bionic study on insects' compound eyes. Optical Technique, 2006, 32: 10-12.

6. J Duparré, F Wippermann. Micro-optical artificial compound eyes. Bioinspiration \& Biomimetics, 2006, 1(1): R1.

7. J Tanida, R Shogenji, Y Kitamura, et al. Color imaging with an integrated compound imaging system. Optics Express, 2003, 11(18): 2109-2117.

8. R Shogenji, Y Kitamura, K Yamada, et al. Bimodal fingerprint capturing system based on compound-eye imaging module. Applied Optics, 2004, 43(6): 1355-1359.

9. R Horisaki, S Irie, Y Ogura, et al. Three-dimensional information acquisition using a compound imaging system. Optical Review, 2007, 14(5): 347-350.

10. M Oikawa, K Iga, T Sanada, et al. Array of distributed-index planar microlenses prepared from ion exchange technique. Japanese Journal of Applied Physics, 1981, 20(4): L296.

11. N F Borrelli, D L Morse, R H Bellman, et al. Photolytic technique for producing microlenses in photosensitive glass. Applied Optics, 1985, 24(16): 2520-2525.

12. Z D Popovic, R A Sprague, G N Connell. Technique for monolithic fabrication of microlens arrays. Applied Optics, 1988, 27(7): 1281-1284.

13. M Kubo, M Hanabusa. Fabrication of microlenses by laser chemical vapor deposition. Applied Optics, 1990, 29(18): 2755-2759.

14. D M Hartmann, O Kibar, S Esener. Polymer microlens arrays fabricated using the hydrophobic effect. Proc. SPIE, 2000: 496-507.

15. Y Fu, B K A Ngoi. Investigation of diffractive-refractive microlens array fabricated by focused ion beam technology. Optical Engineering, 2001 40(4): 511-516.

16. N Ong, Y Koh, Y Q Fu. Microlens array produced using hot embossing process. Microelectronic Engineering, 2002, 60(3): 365-379.

17. P Merz, H J Quenzer, H Bernt, et al. A novel micromachining technology for structuring borosilicate glass substrates. TRANSDUCERS, Solid-State Sensors, Actuators and Microsystems, 12th International Conference on, 2003: 258-261.

18. X Zhang, F Fang, L Yu, et al. Slow slide servo turning of compound eye lens. Optical Engineering, 2013, 52(2): 023401-023401.

19. F Fang, $X$ Zhang, $X$ Hu. Cylindrical coordinate machining of optical freeform surfaces. Optics Express, 2008, 16(10): 7323-7329.

20. X Liu, X Zhang, F Fang, et al. Influence of machining errors on form errors of microlens arrays in ultra-precision turning. International Journal of Machine Tools and Manufacture, 2015, 96: 80-93.
21. D P Yu, G S Hong, Y San Wong. Profile error compensation in fast tool servo diamond turning of micro-structured surfaces. International Journal of Machine Tools and Manufacture, 2012, 52(1): 13-23.

22. D Floreano, R Pericet-Camara, S Viollet, et al. Miniature curved artificial compound eyes. Proceedings of the National Academy of Sciences, 2013, 110(23): 9267-9272.

23. Y M Song, Y Xie, $V$ Malyarchuk, et al. Digital cameras with designs inspired by the arthropod eye. Nature, 2013, 497(7447): 95-99.

24. M K Park, H J Lee, J S Park, et al. Design and fabrication of multi-focusing microlens array with different numerical apertures by using therma reflow method. Journal of the Optical Society of Korea, 2014, 18(1): 71-77.

25. M Levoy, R Ng, A Adams, et al. Light field microscopy. ACM Transactions on Graphics (TOG), 2006, 25(3): 924-934.

26. R Stevens, T Miyashita. Review of standards for microlenses and microlens arrays. The Imaging Science Journal, 2010, 58(4): 202-212.

27. R R Syms, E M Yeatman, V M Bright, et al. Surface tension-powered selfassembly of microstructures-the state-of-the-art. Journal of Microelectromechanical systems, 2003, 12(4): 387-417.

28. H Yang, C K Chao, M KWei, et al. High fill-factor microlens array mold insert fabrication using a thermal reflow process. Journal of Micromechanics and Microengineering, 2004, 14(8): 1197.

29. S Moore, J Gomez, D Lek, et al. Experimental study of polymer microlens fabrication using partial-filling hot embossing technique. Microelectronic Engineering, 2016, 162: 57-62.

30. S Di, H Lin, R Du. An artificial compound eyes imaging system based on mems technology. Robotics and Biomimetics (ROBIO), 2009 IEEE International Conference, 2009: 13-18.

31. D Daly, R Stevens, M Hutley, et al. The manufacture of microlenses by melting photoresist. Measurement Science and Technology, 1990, 1(8): 759.

32. S Haselbeck, H Schreiber, J Schwider, et al. Microlenses fabricated by melting a photoresist on a base layer. Optical Engineering-BellinghamInternational Society for Optical Engineering, 1993, 32: 1322-1322.

33. TR Jay, M B Stern. Preshaping photoresist for refractive microlens fabrication. Opt. Eng., 1994, 33(11): 3552-3555.

34. S Audran, B Faure, B Mortini, et al. Study of dynamical formation and shape of microlenses formed by the reflow method. Proc. SPIE, 2006: 61534D.

35. CY Chang, S Y Yang, L S Huang, et al. Fabrication of polymer microlens arrays using capillary forming with a soft mold of micro-holes array and UV-curable polymer. Optics Express, 2006, 14(13): 6253-6258.

36. C Pan, TWu, M Chen, et al. Hot embossing of micro-lens array on bulk metallic glass. Sensors and Actuators A: Physical, 2008, 141 (2): 422-431.

37. D Yao, P Nagarajan, $L \mathrm{Li}$, et al. A two-station embossing process for rapid fabrication of surface microstructures on thermoplastic polymers. Polymer Engineering \& Science, 2007, 47(4): 530-539.

38. Y Ishii, S Koike, Y Arai, et al. Ink-jet fabrication of polymer microlens for optical-I/O chip packaging. Japanese Journal of Applied Physics, 2000, 39(3S): 1490.

39. Y Luo, L Wang, Y Ding, et al. Direct fabrication of microlens arrays with high numerical aperture by ink-jetting on nanotextured surface. Applied Surface Science, 2013, 279: 36-40.

40. J Y Kim, N B Brauer, V Fakhfouri, et al. Hybrid polymer microlens arrays with high numerical apertures fabricated using simple ink-jet printing technique. Optical Materials Express, 2011, 1(2): 259-269.

41. JY Kim, K Pfeiffer, A Voigt, et al. Directly fabricated multi-scale microlens arrays on a hydrophobic flat surface by a simple ink-jet printing technique. Journal of Materials Chemistry, 2012, 22(7): 3053-3058.

42. X Zhu, L Zhu, H Chen, et al. Fabrication of high numerical aperture microlens array based on drop-on-demand generating of water-based molds. Optics \& Laser Technology, 2015, 68: 23-27.

43. J Albero, L Nieradko, C Gorecki, et al. Fabrication of spherical microlenses by a combination of isotropic wet etching of silicon and molding techniques. Optics Express, 2009, 17(8): 6283-6292.

44. R Bitterli, T Scharf, H-P Herzig, et al. Fabrication and characterization of linear diffusers based on concave micro lens arrays. Optics Express, 2010, 18(13): 14251-14261.

45. G Du, Q Yang, F Chen, et al. Direct fabrication of seamless roller molds with gapless and shaped-controlled concave microlens arrays. Optics Letters, 2012, 37(21): 4404-4406.

46. X Meng, F Chen, Q Yang, et al. Simple fabrication of closed-packed IR microlens arrays on silicon by femtosecond laser wet etching. Applied Physics A, 2015, 121(1): 157-162. 
47. $X J Y i, X Y$ Zhang, $Y$ Li, et al. Microlens arrays formed by melting photoresist and ion beam milling. Proc. SPIE,1998: 249-253.

48. L Harriott, R Scotti, K Cummings, et al. Micromachining of integrated optical structures. Applied Physics Letters, 1986, 48(25): 1704-1706.

49. E B Kley, T Possner, R Göring. Realization of micro-optic and integrated optic components by electron-beam-lithographic surface profiling and ion exchange in glass. International Journal of Optoelectronnics, 1993, 8: 513-513.

50. T Fujita, H Nishihara, J Koyama. Fabrication of micro lenses using electronbeam lithography. Optics Letters, 1981, 6(12): 613-615.

51. A Brückner, J Duparré, P Dannberg, et al. Artificial neural superposition eye. Optics Express, 2007, 15(19): 11922-11933.

52. KH Jeong, J Kim, L P Lee. Biologically inspired artificial compound eyes. Science, 2006, 312(5773): 557-561.

53. H Yu, G Zhou, F S Chau, et al. Fabrication and characterization of PDMS microlenses based on elastomeric molding technology. Optics Letters, 2009, 34(21): 3454-3456.

54. KWei, H Zeng, Y Zhao. Insect-Human Hybrid Eye (IHHE): an adaptive optofluidic lens combining the structural characteristics of insect and human eyes. Lab on a Chip, 2014, 14(18): 3594-3602.

55. M Wang, TWang, $\mathrm{H}$ Shen, et al. Subtle control on hierarchic reflow for the simple and massive fabrication of biomimetic compound eye arrays in polymers for imaging at a large field of view. Journal of Materials Chemistry C, 2016, 4(1): 108-112.

56. E Brinksmeier, $L$ Autschbach. Ball-end milling of free-form surfaces for optical mold inserts. Proceedings of 19th Annual Meeting of American Society for Precision Engineering (ASPE), 2004: 88-91.

57. J Yan, Z Zhang, T Kuriyagawa, et al. Fabricating micro-structured surface by using single-crystalline diamond endmill. The International Journal of Advanced Manufacturing Technology, 2010, 51(9): 957-964.

58. A Yi and L Li. Design and fabrication of a microlens array by use of a slow tool servo. Optics Letters, 2005, 30(13): 1707-1709.

59. J Zhou, L Li, N Naples, et al. Fabrication of continuous diffractive optical elements using a fast tool servo diamond turning process. Journal of Micromechanics and Microengineering, 2013, 23(7): 075010.
60. F Z Chen, C H Chen, C H Wu, et al. Development of a double-sided micro lens array for micro laser projector application. Optical Review, 2012, 19(4): 238-241.

61. CY Chan, L H Li, W B Lee, et al. Research on light field imaging based on compound eye ultra-precision machining. Journal of Mechanical Engineering, 2016, 52(17): 50-57.

62. ZW Zhu, STo, W L Zhu, et al. Feasibility study of the novel quasi-elliptical tool servo for vibration suppression in the turning of micro-lens arrays. International Journal of Machine Tools and Manufacture, 2017, 122: 98-105.

63. L Li, Y Y Allen. Development of a 3D artificial compound eye. Optics Express, 2010, 18(17): 18125-18137.

64. L Li, YY Allen. Microfabrication on a curved surface using 3D microlens array projection. Journal of Micromechanics and Microengineering, 2009, 19(10): 105010.

65. M Levoy, P Hanrahan. Light field rendering. Proceedings of the 23rd Annual Conference on Computer Graphics and Interactive Techniques,1996: 31-42.

66. E H Adelson, J Y Wang. Single lens stereo with a plenoptic camera. IEEE Transactions on Pattern Analysis and Machine Intelligence, 1992, 14(2): 99-106

67. B Wilburn, M Smulski, H-H K Lee, et al. The light field video camera. Media Processors 2002, 2002, 4674: 29-36.

68. B Wilburn, N Joshi, V Vaish, et al. High performance imaging using large camera arrays. ACM Transactions on Graphics (TOG), 2005: 765-776.

69. T Georgiev, C Intwala. Light field camera design for integral view photography. Adobe Technical Report, 2006.

70. R Ng, M Levoy, M Brédif, et al. Light field photography with a hand-held plenoptic camera. Computer Science Technical Report CSTR, 2005, 2(11): $1-11$.

71. MW Tao, S Hadap, J Malik, et al. Depth from combining defocus and correspondence using light-field cameras. Proceedings of the IEEE International Conference on Computer Vision, 2013: 673-680.

72. R Ueno, K Suzuki, M Kobayashi, et al. Compound-eye camera module as small as $8.5 \$ \backslash$ times $\$ 8.5 \$$ times $\$ 6.0 \mathrm{~mm}$ for $26 \mathrm{k}$-Resolution Depth Map and 2-Mpix 2D Imaging. IEEE Photonics Journal, 2013, 5(4): 6801212-6801212.

\section{Submit your manuscript to a SpringerOpen ${ }^{\circ}$ journal and benefit from:}

- Convenient online submission

- Rigorous peer review

- Open access: articles freely available online

- High visibility within the field

- Retaining the copyright to your article

Submit your next manuscript at $\boldsymbol{\nabla}$ springeropen.com 